\title{
A GAUSSIAN PROCESS BASED MULTI-PERSON INTERACTION MODEL
}

\author{
T. Klinger, F. Rottensteiner, C. Heipke
}

Institute of Photogrammetry and GeoInformation, Leibniz Universität Hannover, Germany -

(klinger, rottensteiner, heipke)@ipi.uni-hannover.de

\author{
Commission III, WG III/3
}

KEY WORDS: Gaussian Processes, Interactions, Online, Pedestrians, Tracking, Video

\begin{abstract}
:
Online multi-person tracking in image sequences is commonly guided by recursive filters, whose predictive models define the expected positions of future states. When a predictive model deviates too much from the true motion of a pedestrian, which is often the case in crowded scenes due to unpredicted accelerations, the data association is prone to fail. In this paper we propose a novel predictive model on the basis of Gaussian Process Regression. The model takes into account the motion of every tracked pedestrian in the scene and the prediction is executed with respect to the velocities of all interrelated persons. As shown by the experiments, the model is capable of yielding more plausible predictions even in the presence of mutual occlusions or missing measurements. The approach is evaluated on a publicly available benchmark and outperforms other state-of-the-art trackers.
\end{abstract}

\section{INTRODUCTION}

Visual pedestrian tracking is one of the most active research topics in the fields of image sequence analysis and computer vision. The generated trajectories carry important information for the semantic analysis of scenes and thus are a crucial input to many applications in fields such as autonomous driving, fieldrobotics and visual surveillance.

Most available systems for tracking use variants of the recursive Bayes Filter such as the Kalman- or the Particle Filter to find a compromise between image-based measurements (i.e., automatic pedestrian detections) and a motion model. Generally, the motion model is a realisation of a first-order Markov chain which considers the expected dynamic behaviour such as constant velocity or smooth motion. In the absence of measurements, e.g. during an occlusion, the trajectory is continued only by the motion model. For longer intervals of occlusions, however, a first-order Markov chain is prone to drift away from the actual target position. This situation often occurs in crowded environments, where mutual occlusions cannot be avoided and pedestrian movements often do not match the assumption of an un-accelerated motion. Some authors explain the deviations from a constant-velocity model as an effect of social forces caused by interactions with other members of a scene (Helbing and Molnár, 1995). The information gathered from other scene members with respect to motion is often referred to as motion context. In the scope of recursive filtering, this motion context carries valuable information for tracking and allows to generate more plausible predictions in the absence of measurements. In turn, more realistic predictions lower the risk of tracking errors such as false negative detections and identity switches. Available context-aware approaches to pedestrian tracking often require binary decisions about group membership of individuals, or they constrain the interactions by a Markovian assumption. In either way, possible correlations between pedestrians that are not related by the model are discarded.

Our approach considers the context between every possible pair of pedestrians without being explicit about their interactions. To this end we propose a new model for the predictive function of a recursive filter that is based on Gaussian Process Regression. In this context, we formulate a new covariance function taking

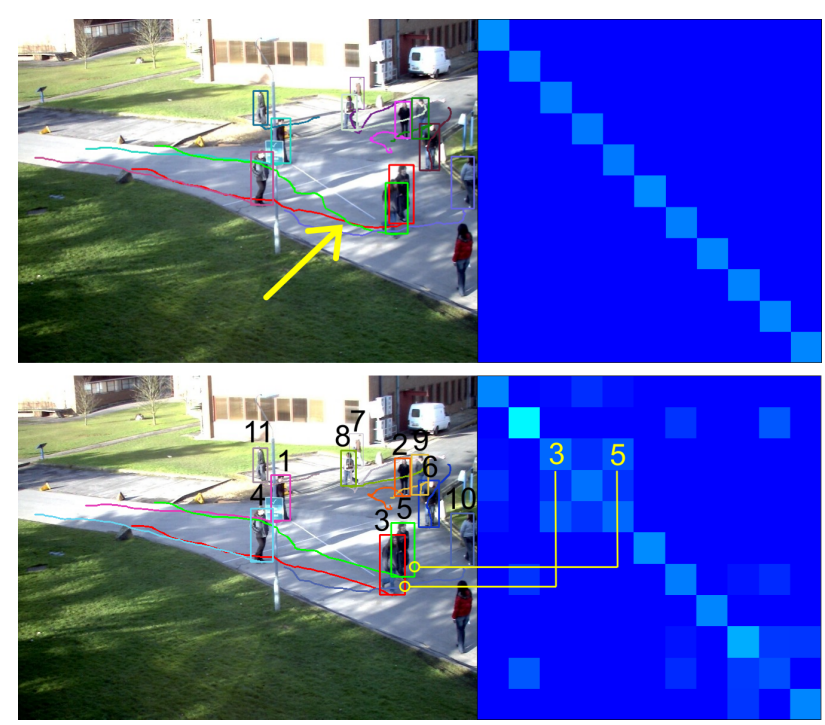

Figure 1. Trajectories generated using a stand-alone Kalman Filter (upper image) and the proposed method (lower image). On the right side of the images the covariance matrices are shown for that scene. The covariance matrix has one row and column for every tracked person in ascending order of their associated identification number. Brighter values indicate higher covariances of the trajectories. Using the stand-alone Kalman Filter, an identity switch occurred after a phase of mutual occlusion (indicated by the arrow), which can be avoided when using the proposed method.

into account the spatial distance and angular displacement of two motion trajectories. The output of the covariance function is used as a measure for the interactions between pedestrians. The covariance matrix stores the covariances of all pairs of pedestrians and is updated at every time step; cf. Fig. 1 for an example. Fig. 1 further shows a situation where an identity switch occurs when using a stand-alone Kalman Filter for the prediction, which can be avoided when using the proposed method. As we avoid explicit grouping of pedestrians, we refer to the information captured by the covariance matrix as implicit motion context . 
The contributions of this paper are the proposal and comprehensive investigation of a new strategy for the incorporation of motion context into a recursive filter. The proposed method is implemented in the framework of a recursive tracking-by-detection framework based on (Klinger et al., 2015). A quantitative evaluation of the results is carried out on the basis of the Multi Object Tracking Benchmark (Leal-Taixé et al., 2015).

The remainder of this paper is structured as follows: First we review the related work on the topic of visual multi-pedestrian tracking with a focus on approaches that investigate motion context, and on the topic of Gaussian Process Regression applied in the context of tracking (Sec. 2). Then, we investigate Gaussian Process Regression in some more detail. Subsequently, we formulate our novel approach (Sec. 3). In the experiments (Sec. 4) we analyse the sensitivity of the proposed method to the variation of the involved parameters and compare the final results with methods from related work on the basis of a publicly available benchmark dataset. In Sec. 5 we conclude our work and give an outlook.

\section{RELATED WORK}

In this section we briefly review related approaches to contextaware multi-person tracking. We further refer to papers that deal with Gaussian Process Regression in the context of recursive filtering approaches.

Motion context. While the main factors for path planning of a pedestrian are well understood (Helbing and Molnár, 1995), there are many approaches for embedding this contextual information into a tracking framework. In (Scovanner and Tappen, 2009) and (Milan et al., 2014) trajectory estimation is formulated as an energy minimisation problem, where the energy is the sum of various terms penalising a deviation from an expected behaviour such as collision avoidance, moving towards a predefined destination in a straight line and constant velocity. Being aware of other pedestrians' positions in the scene, such a motion model considers context to avoid collisions, but possible correlations of the trajectories that indicate mutual patterns of motion are not further evaluated. Ge et al. (2009), Yamaguchi et al. (2011), Pellegrini et al. (2010), Leal-Taixé et al. (2011) and Zhang and van der Maaten (2013) incorporate group models which basically have a smoothing effect on the motion of pedestrians of the same group. Although contextual information w.r.t. the motion of interacting pedestrians is considered in this way, the binary group membership represented by the model neglects potential correlations between subjects from different groups.

Pellegrini et al. (2009), Choi and Savarese (2010), and Yoon et al. (2015) do not apply grouping explicitly. They predict the position of each subject based on the history of all pedestrians. Pellegrini et al. (2009) directly incorporate interactions as well as expected destinations in the scene into the dynamic model of a recursive filter. The degree of interaction between two pedestrians is evaluated by their current distance and by the angular displacement of their trajectories, i.e., the cosine of the angle between their directions of motion. Choi and Savarese (2010) use a Markov Random Field, where the current state estimate is conditioned on the previous one and undirected edges are established between neighbouring subjects, modelling the social forces caused by interactions. However, due to the Markov property interactions of pedestrians which are not direct neighbours in object space are suppressed. As a consequence, potential correlations between subjects that are further apart are neglected. Yoon et al. (2015) also consider the relative motion between subjects by conditioning the current state estimate on the previous state estimate of the same subject and on the subjects in its vicinity. In this way, the motion of different interrelated persons is taken into account, but uncertainties about the previous state estimates are not considered in the estimation of the current state estimates.

Gaussian Process Regression. Gaussian Process (GP) Regression models are well studied in the fields of geodesy (where an equivalent to GP Regression is known as collocation) (Moritz, 1973), geo-statistics (where it is also known as kriging), (Krige, 1951), machine learning (Rasmussen, 2006) and computer vision (e.g. Urtasun et al., 2006). We stick to the terminology of 'Gaussian Processes' as our work is set in the context of photogrammetry and computer vision.

A Gaussian Process is a stochastic process in which any finite subset of random variables has a Gaussian joint distribution. Given a set of input points and observed target variables, a prediction of the target variable for a test point is made by conditioning its target variable on the observed target variables. In this way, the predictive function need not be modelled parametrically; it is merely assumed that the input data are correlated. Each conditional distribution over a target variable is Gaussian, which favours their application in the context of recursive Bayes Filters. Ko and Fox (2009) use GPs for Bayesian filtering (GPBayes Filter), emphasising that in this way parametric prediction and parametric observation models can be avoided. For the prediction of a robot's state transition, the authors define a Gaussian Process taking as input previous state and control sequences of the robot. Ellis et al. (2009) applied GP-Bayes Filters to the tracking of pedestrians, where the input data are trajectories of different persons observed in the past. The problem is formulated as a regression task, where velocities are estimated on the basis of the previously observed trajectories. For a predictive model which is representative for a complete scene, a high amount of training data may be required (depending on the complexity of the scene). As the trajectories are required a priori, the application is restricted to offline processing. Kim et al. (2011) apply GP based regression for the prediction of motion trajectories of vehicles. Individual trajectories are assigned to clusters and outliers are detected when the trajectories deviate from a so-called mean flow field. By the explicit association of the trajectories to clusters, possible correlations between trajectories from different clusters are not considered. Later the same authors applied GP regression to detect regions of interest for camera orientation, when acquiring images of a football match, by looking at the means of the regression model which reflect the expected destinations of the involved subjects (football players) (Kim et al., 2012). Here, the motion trajectories are not regarded further and persons are correlated based on the spatial distance only. In these works, the input data are the 2D locations of the subjects and the target variables are their velocities.

Most of the cited papers dealing with motion context are either explicit about the grouping of pedestrians, so that possible correlations among members of different groups are ignored, or they limit the range of related objects by a Markov assumption. The related work on Gaussian Process Regression in the context of tracking does not include any approach to the tracking of pedestrians in which the interactions are estimated together with the trajectories at runtime. We adapt the work of Pellegrini et al. (2009) by refining the predictive model of a recursive filter so that it takes into account all of the other scene members. Different from Pellegrini et al. (2009), we apply a predictive function on the basis of Gaussian Processes, mitigating the need for a parametric prediction model. In this respect, our approach is related to the works of Ellis et al. (2009) and Kim et al. (2012) in the way of predicting the velocity, with the key difference that we assess 
the similarity between trajectories at runtime. In this way, we directly incorporate contextual information into the tracking framework and simultaneously estimate the velocities together with the interactions. This enables more reliable predictions even in the absence of measurements.

\section{METHOD}

In this section we explain the principles of Gaussian Process Regression (GPR) and formulate the prediction model of a recursive filter as a GPR problem. To make the prediction sensitive to interactions, we propose a new covariance function, which takes a pair of motion trajectories as input and computes a measure of similarity based on the motion direction and the spatial distance of the trajectories. We assume that pedestrians interact to the degree of the covariance of their motion trajectories.

\subsection{Gaussian Process Regression}

In a Gaussian Process Regression model, it is assumed that the function values of a target variable $y$ are drawn from a noisy process:

$$
y=f(x)+n
$$

with Gaussian white noise $n$ with variance $\sigma_{n}^{2}$. The regression function $f(x)=t(x)+s$ is composed of a deterministic part $t(x)$, also referred to as the trend, and a stochastic part $s \sim$ $\mathcal{N}\left(0, \Sigma_{s s}\right)$, which is referred to as the signal and which follows a zero-mean normal distribution with covariance $\Sigma_{s s}$. It is further assumed that the signal at close positions is correlated. Given a set of observed input and target variables $\mathcal{D}=\left\{\left(x_{1}, y_{1}\right), \ldots,\left(x_{n}, y_{n}\right)\right\}$, the aim is to predict the target variable $y_{*}$ for a new input point $x_{*}$. By definition, any finite subset of the random variables in a GP has a Gaussian joint distribution (Rasmussen, 2006). Hence the joint distribution of an unknown target variable $y_{*}$ and the observed data $\mathbf{y}=\left\{y_{1}, \ldots, y_{n}\right\}$ is Gaussian and can be modelled according to Eq. 1,

$$
\left[\begin{array}{c}
\mathbf{y} \\
y_{*}
\end{array}\right] \sim \mathcal{N}\left(\left[\begin{array}{c}
E(\mathbf{y}) \\
E\left(y_{*}\right)
\end{array}\right],\left[\begin{array}{cc}
K & K_{*}^{T} \\
K_{*} & K_{* *}
\end{array}\right]\right)
$$

where $E(\mathbf{y})$ and $E\left(y_{*}\right)$ are the expected values of the observed and the unknown target variables that correspond to the trend. The covariance matrix is a block matrix, whose elements are specified by a covariance function $k\left(x_{i}, x_{j}\right)$. The matrix $K$ is the covariance matrix of the observed target variables, such that $K(i, j)=k\left(x_{i}, x_{j}\right), K_{*}$ is the vector of covariances of the observed and the unknown target variables, such that $K_{*}(i)=$ $k\left(x_{*}, x_{i}\right)$, and $K_{* *}=k\left(x_{*}, x_{*}\right)$ is the variance of the unknown target variable. One of the most prominent realisations of the covariance function is the squared exponential or Gaussian function (Rasmussen, 2006).

$$
k\left(x_{i}, x_{j}\right)=\sigma_{f}^{2} \cdot \exp \left(-\frac{\left(x_{i}-x_{j}\right)^{2}}{2 l^{2}}\right)+\sigma_{n}^{2} \cdot \delta(i=j)
$$

Eq. 2 describes a Gaussian covariance function, where the covariance of two input points $x_{i}$ and $x_{j}$ is dependent on their pairwise distance with decreasing covariance at growing distances. The signal variance $\sigma_{f}^{2}$ basically controls the uncertainty of predictions at input points far from observed data. The characteristic length-scale $l$ controls the range of correlations in the input space. In Eq. $2 \sigma_{n}^{2}$ is the noise variance accounted for in the diagonal elements of $K$, and $\delta(i=j)$ is the Kronecker delta function, which is 1 for $i=j$ and 0 otherwise.

The prediction of a new target variable for the input point $x_{*}$ is made by building the conditional distribution of the desired target variable $y_{*}$ based on Eq. 1. Since the distribution in Eq. 1 is Gaussian, the same holds true for the conditional probability of the unknown target variable. In a Gaussian Process Regression model, the distribution over the predicted target variable thus can be written as Eq. 3,

$$
P\left(y_{*} \mid \mathbf{y}\right) \sim \mathcal{N}\left(\mathcal{G P}_{\mu}\left(x_{*}, \mathcal{D}\right), \mathcal{G} \mathcal{P}_{\Sigma}\left(x_{*}, \mathcal{D}\right)\right),
$$

with

$$
\mathcal{G} \mathcal{P}_{\mu}\left(x_{*}, \mathcal{D}\right)=E\left(y_{*}\right)+K_{*} K^{-1}(\mathbf{y}-E(\mathbf{y})),
$$

and

$$
\mathcal{G} \mathcal{P}_{\Sigma}\left(x_{*}, \mathcal{D}\right)=K_{* *}-K_{*} K^{-1} K_{*}^{T} .
$$

The estimated value $\hat{y}_{*}=\mathcal{G} \mathcal{P}_{\mu}\left(x_{*}, \mathcal{D}\right)$ corresponds to the mean of Eq. 3, and $\hat{\sigma}_{y_{*}}^{2}=\mathcal{G} \mathcal{P}_{\Sigma}\left(x_{*}, \mathcal{D}\right)$ is the variance of the estimated target variable.

\subsection{Implicit Motion Context}

For the application in a recursive filter, the aim is to formulate a predictive model which takes account of the assumption that pedestrians do not move in a way completely independent from other scene members. Similar to Ellis et al. (2009) and Kim et al. (2011) we model the velocity $v_{i}$ as the target variable of a GP Regression model independently for each input dimension. Different from the related work we take as input the trajectories and velocities of all currently tracked pedestrians. In accordance with the GPR model we decompose the velocity of a pedestrian into a trend and a signal, and argue that the signal of two pedestrians is correlated in case of interactions. In analogy to Eq. 3 the predictive model for the velocity can be written in probabilistic form as a Gaussian distribution over the predicted velocity:

$$
P\left(v_{i} \mid \mathbf{v}\right) \sim \mathcal{N}\left(\mathcal{G P}{ }_{\mu}\left(\mathcal{T}_{i}, \mathbf{T}\right), \mathcal{G} \mathcal{P}_{\Sigma}\left(\mathcal{T}_{i}, \mathbf{T}\right)\right),
$$

where the given set of input and target variables $\mathbf{T}=\left\{\left(\mathcal{T}_{1}, v_{1}\right), \ldots,\left(\mathcal{T}_{n}, v_{n}\right)\right\}$ consists of the trajectories and current velocity estimates of all $n$ currently tracked pedestrians. The predicted velocity of a person $i$ can be found using Eqs. 4 and 5. We propose a novel covariance function (Eq. 7) that computes the covariance of two trajectories $\mathcal{T}_{i}=\left[\mathbf{X}_{i, t-h}, \ldots, \mathbf{X}_{i, t}\right]^{T}$ and $\mathcal{T}_{j}=\left[\mathbf{X}_{j, t-h}, \ldots, \mathbf{X}_{j, t}\right]^{T}$ w.r.t. their current and $h$ most recent positions in object space. We assume that the motion direction and spatial distance of two pedestrians are representative for their interactions. This is why the function takes into account the angular displacement of the motion directions and the spatial distance between the current positions.

$k\left(\mathcal{T}_{i}, \mathcal{T}_{j}\right)=w\left(\mathcal{T}_{i}, \mathcal{T}_{j}\right) \sigma_{f}^{2} \exp \left(-\frac{\left(\mathbf{X}_{i, t}-\mathbf{X}_{j, t}\right)^{2}}{2 l^{2}}\right)+\sigma_{n, i}^{2} \delta(i=j)$

The first factor in Eq. 7, $w\left(\mathcal{T}_{i}, \mathcal{T}_{j}\right)$, is what we define as the $a n$ gular function which takes into account the angular displacement $\alpha_{i j}$ of two trajectories (see Eq. 8). The noise variance $\sigma_{n, i}^{2}$ reflects the uncertainty about the input velocities and is added along the diagonal of $K$. We compute $\alpha_{i j}$ as the angle between the connecting lines of the first and last points of $\mathcal{T}_{i}$ and $\mathcal{T}_{j}$, respectively. The function returns the cosine of the angular displacement if the angular displacement is smaller than a threshold $\alpha_{0}$, and otherwise it is set to zero.

$$
w\left(\mathcal{T}_{i}, \mathcal{T}_{j}\right)=\cos \left(\alpha_{i j}\right) \cdot \delta\left(\alpha_{i j} \leq \alpha_{0}\right)
$$

The signal variance $\sigma_{f}^{2}$, the length-scale $l$, the history $h$ and $\alpha_{0}$ are parameters whose optimal values are determined in the experiments. Fig. 2 shows the principle of the angular function. The velocity vectors of four fictitious trajectories are visualised by ar- 

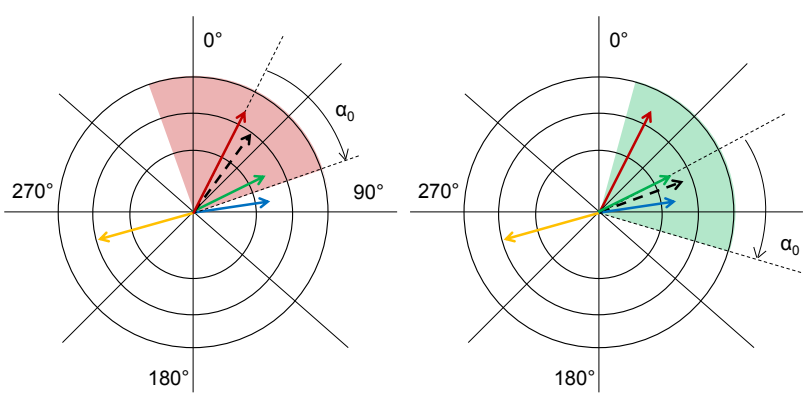

Figure 2. Angular displacements and weighted average velocities (see text for details).

rows in different colours, and the shaded regions show the ranges of possible correlations for the red (left) and the green (right) instance. These ranges are determined by the angular threshold $\alpha_{0}$, and all velocities inside the shaded region contribute to the estimated velocity. For a tracking in $2 \mathrm{D}$, the weighted average velocity vector $\overline{\mathbf{v}}_{i}$ is drawn by the broken arrow in Fig. 2. We model $\overline{\mathbf{v}}_{i}$ as the trend of the Gaussian Process in accordance with Eq. 9.

$$
\overline{\mathbf{v}}_{i}=E\left(\mathbf{v}_{i}\right)=\frac{1}{n} \sum_{j=1 \ldots n} w\left(\mathcal{T}_{i}, \mathcal{T}_{j}\right) \cdot \mathbf{v}_{j}
$$

The exponential function on the r.h.s. of Eq. 7 accounts for the spatial distance between the current position estimates $\mathbf{x}_{i, t}$ and $\mathbf{x}_{j, t}$. Fig. 3 shows velocity estimates computed using the exponential function for all points in a discrete grid of $0.5 \mathrm{~m} \times 0.5 \mathrm{~m}$, using the current positions of the persons as input variables and their velocities as observed target variables. The variances of the predicted velocities are indicated by the colours of the arrows, where red indicates high values and green indicates low values. Note that due to the exponential decrease of the covariances the predicted velocities for positions with a low density of observed data have higher variances (depending on $\sigma_{f}^{2}$ ), and the estimated velocities in these areas tend to zero. While in Eq. 7 the exponential function causes high covariances for all pedestrians at short pairwise distances (depending on $l$ ), the delta function suppresses correlations of pedestrians moving in different directions (depending on the angular displacement threshold $\alpha_{0}$ ).

Having defined the covariance function in Eq. 7, the covariance is computed for every pair of currently tracked pedestrians. Given the observed input data $\mathbf{T}$, the predicted values of the velocity components $\hat{v}_{i}$ and their variances $\hat{\sigma}_{v i}^{2}$ are found in accordance with Eqs. 4 and 5, respectively:

$$
\begin{gathered}
\hat{v}_{i}=\bar{v}_{i}+K_{*} K^{-1}(\mathbf{v}-E(\mathbf{v})), \\
\hat{\sigma}_{v i}^{2}=K_{* *}-K_{*} K^{-1} K_{*}^{T} .
\end{gathered}
$$

\subsection{Recursive Bayesian estimation}

For the practical application of the proposed model in a recursive filter we use an Extended Kalman Filter model. Generally, a recursive Bayesian estimation framework consists of a predictive model for the transition of the state vector $\mathbf{w}$ between successive time steps, and a measurement model, which yields a mapping from the state space to the observation space (here: the image). We model a six-dimensional state vector that accounts for the 3D position $(X, Y, Z)$, the height $H$ and the velocity components $v_{X}$ and $v_{Z}$ in the dimensions $X$ and $Z$ parallel to the ground plane, which is assumed to be a plane at a known and constant height below the camera.

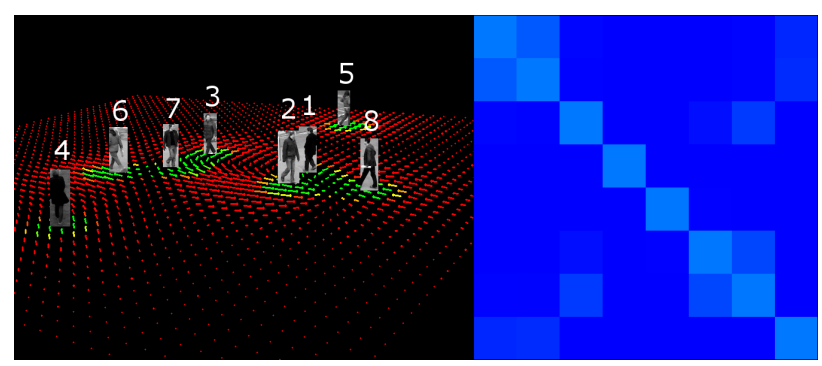

Figure 3. Motion vector field with covariances as a function of the distance only. The magnitudes of the velocities are exaggerated by a factor of two for visualisation.

Prediction. One Gaussian Process is defined independently for the velocity $v_{X}$ and $v_{Z}$,

$$
v_{X i} \sim \mathcal{N}\left(\mathcal{G} \mathcal{P}_{\mu_{X}}\left(\mathcal{T}_{i}, \mathbf{T}\right), \mathcal{G} \mathcal{P}_{\Sigma_{X}}\left(\mathcal{T}_{i}, \mathbf{T}\right)\right)
$$

and

$$
v_{Z i} \sim \mathcal{N}\left(\mathcal{G} \mathcal{P}_{\mu_{Z}}\left(\mathcal{T}_{i}, \mathbf{T}\right), \mathcal{G} \mathcal{P}_{\Sigma_{Z}}\left(\mathcal{T}_{i}, \mathbf{T}\right)\right)
$$

so that the prediction of the velocities is accomplished by finding the means of the univariate Gaussian distributions in accordance with Eqs. 10 and 11. The predictive model of the recursive filter is a multivariate Gaussian distribution over the state vector, Eq. 14 , conditioned on the previous state vectors $\mathbf{w}_{t-1, i=1 \ldots n}$ and trajectories $\mathcal{T}_{i=1 \ldots n}$ of all $n$ pedestrians being tracked:

$$
P\left(\mathbf{w}_{t, i}^{+} \mid \mathbf{w}_{t-1, i=1 \ldots n}, \mathcal{T}_{i=1 \ldots n}\right)=\mathcal{N}\left(\mu_{w, t}^{+}, \Sigma_{w w, t}^{+}\right),
$$

with mean vector $\mu_{w, t}^{+}$:

$$
\mu_{w, t}^{+}=\left[X_{t}^{+}, Y_{t}^{+}, Z_{t}^{+}, H_{t}^{+}, v_{X, t}^{+}, v_{Z, t}^{+}\right]^{T}
$$

and covariance matrix $\Sigma_{w w, t}^{+}$:

$$
\Sigma_{w w, t}^{+}=\Psi \Sigma_{w w, t-1} \Psi^{T}+\Sigma_{p},
$$

where $\Psi$ is the transition matrix that transforms the state vector from the previous time step to the current time step (Rabiner, 1989). We assume zero acceleration in the directions of $X$ and $Z$ and zero velocity in vertical direction, i.e., for the parameters $Y$ and $H$. Deviation from these assumptions may occur due to unforeseen accelerations $\left(a_{X}, a_{Z}\right)$ and velocities $\left(v_{Y}, v_{H}\right)$. These effects are captured by a zero-mean multivariate normal distribution over the vector $\mathbf{u}=\left[a_{X}, v_{Y}, a_{Z}, v_{H}\right]^{T}$ with expectation $E(\mathbf{u})=\mathbf{0}$ and covariance $\Sigma_{u u}=\operatorname{diag}\left(\sigma_{a X}^{2}, \sigma_{v Y}^{2}, \sigma_{a Z}^{2}, \sigma_{v H}^{2}\right)$. These uncertainties are related to the covariance of the predicted state vector by the process noise $\Sigma_{p}=G \Sigma_{u u} G^{T}$, where the matrix $G$ is the Jacobian of the transition matrix. In our case the accelerations are induced by interactions with other pedestrians, so that the variances computed by the Gaussian Process Regression reflect the uncertainties about the process noise, i.e., $\sigma_{a X}^{2}=\mathcal{G} \mathcal{P}_{\Sigma_{X}}\left(\mathcal{T}_{i}, \mathbf{T}\right) \cdot \Delta t^{-2}$ and $\sigma_{a Z}^{2}=\mathcal{G} \mathcal{P}_{\Sigma_{Z}}\left(\mathcal{T}_{i}, \mathbf{T}\right) \cdot \Delta t^{-2}$, where $\Delta t$ is the time difference between two consecutive frames. Note that the noise variances $\sigma_{n, i}^{2}$, which reflect the uncertainties about the observed target variables in Eq. 7, are the posterior variances of the velocities from the previous time step. Incorporating the estimated velocities into the predicted state vector yields the following functional model of the prediction:

Position:

$$
\begin{aligned}
X_{t}^{+} & =X_{t-1}+v_{X, t}^{+} \Delta t \\
Z_{t}^{+} & =Z_{t-1}+v_{Z, t}^{+} \Delta t
\end{aligned}
$$

Height:

$$
Y_{t}^{+}=Y_{t-1}
$$



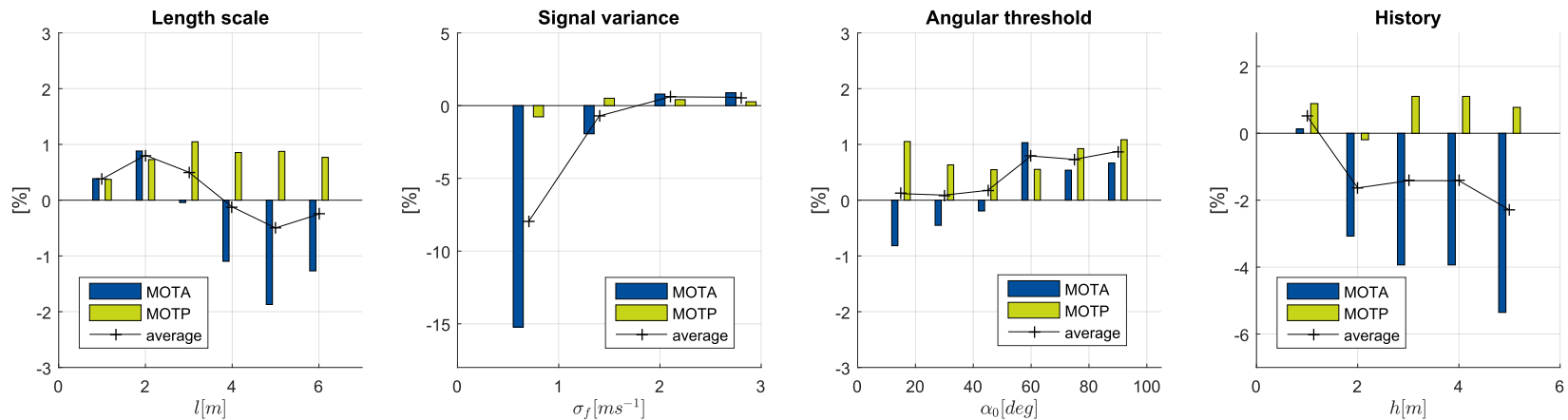

Figure 4. Variation of the parameters and difference of the sum of MOTA and MOTP metrics relative to the results from the stand-alone Kalman Filter.

$$
H_{t}^{+}=H_{t-1}
$$

Velocity:

$$
\begin{aligned}
& v_{X, t}^{+}=\hat{v}_{X *} \\
& v_{Z, t}^{+}=\hat{v}_{Z *}
\end{aligned}
$$

Update. As in Klinger et al. (2015), we apply a Dynamic Bayesian Network (DBN) which combines the results of a pedestrian detector, an instance specific classifier with online training capability and a Kalman Filter model in a single probabilistic tracking-by-detection framework. The update step of the recursive filter is guided by the collinearity equations. The results of the pedestrian detector and the instance specific classifier are modelled as observations of the positions of the feet and the head of the pedestrians in the image. We apply a pedestrian detector on the basis of the HOG/SVM (Dalal and Triggs, 2005) and an instance specific classifier on the basis of Online Random Forests (Saffari et al., 2009). We further use a fictitious observation for the height of the feet, which is identical to the height of the ground plane. This observation basically enables the conversion of 2D image coordinates to $3 \mathrm{D}$ world coordinates and, thus, tracking in 3D space.

\section{EXPERIMENTS}

The covariance function we proposed in Sec. 3. depends on four different parameters: The signal variance $\sigma_{f}^{2}$, the length-scale $l$, the angular threshold $\alpha_{0}$ and the history of recent trajectory points $h$. For practical application, $\alpha_{0}, h, \sigma_{f}$ and $l$ are learnt from the training data of the 3DMOT benchmark dataset (LealTaixé et al., 2015) using the direct search approach (Hooke and Jeeves, 1961). In a first experiment we demonstrate the sensitivity of the tracking results w.r.t. the mentioned parameters. We furthermore evaluate our approach against methods from related work on the basis of the 3DMOT benchmark.

\subsection{Training of the parameters}

For the determination of the parameters $\mathbf{p}=\left\{\alpha_{0}, h, \sigma_{f}, l\right\}$ that yield optimal results on the training data, we take the argument variables $\hat{\mathbf{p}}$ that solve the minimisation problem

$$
\hat{\mathbf{p}}=\underset{\mathbf{p}}{\operatorname{argmin}} 1-\frac{\operatorname{MOTA}(\mathbf{p})+\operatorname{MOTP}(\mathbf{p})}{2} .
$$

We applied the direct search approach and changed one parameter at a time, keeping the others fixed. The parameters yielding the best results are kept fix during the variation of the next parameter. We assume independence of the parameters and do not further iterate this procedure. As all evaluation metrics used in the experiments, except for the runtime, essentially depend on $\mathbf{p}$, the notation with $\mathbf{p}$ as function argument will be avoided in the remainder of this paper. MOTA and MOTP are the classification of events, activities, and relationships (CLEAR) metrics Multiple Object Tracking Accuracy and Multiple Object Tracking Precision as defined by Bernardin and Stiefelhagen (2008). The MOTA metric takes into account false positive (FP) and false negative (FN) assignments as well as identity switches (IDs). The MOTP metric reflects the geometric accuracy of the tracking results. A detection is counted as correct if the estimated position of the feet is not more than one metre apart from the reference. In Fig. 4 the results of the training procedure are visualised. The Figure is divided into four parts, each showing the results achieved by the variation of one parameter. The results are drawn relative to the results achieved by not using the proposed method, that is, by using a stand-alone Kalman Filter instead. The curve shows the average gain or loss of MOTA and MOTP achieved by using the proposed method (the connecting lines are just drawn for visual support). The parameters associated to the peaks of the average curves are taken as optimal values.

We determined values of $l=2 m, \sigma_{f}=2 \mathrm{~m} / \mathrm{s}, \alpha_{0}=90^{\circ}$ and $h=1 m$ to yield optimal results. The length-scale parameter indicates that interactions take place basically in a radius of $2 m$ around a person. The signal variance $\sigma_{f}^{2}$ controls the maximum range of velocities and basically limits the velocity estimates far from the input data. The value achieved by the training thus indicates that $68 \%$ of the velocities are expected to lie in a range of $\pm 2 \mathrm{~m} / \mathrm{s}$. The angular threshold of $90^{\circ}$ indicates that considering all persons moving with an angular displacement of at most $90^{\circ}$ positively affects the tracking results. The history of $1 \mathrm{~m}$ indicates that only the last metre of the trajectories contributes to the tracking; if longer parts of the trajectories are taken into account, sudden changes in the direction of motion do not reflect instantaneously in the covariances of the trajectories, and the performance decreases.

\subsection{DMOT challenge}

Here, we report results achieved on the 3DMOT benchmark dataset (Leal-Taixé et al., 2015) using our approach. For the initialisation of new trajectories we only use the automatic detection results provided along with the benchmark dataset. Our results and results from Pellegrini et al. (2009), Leal-Taixé et al. (2011), Klinger et al. (2015) and from a baseline tracker are available online $^{1}$. The test dataset consists of two image sequences, the PETS09-S2L2 sequence from a campus and the AVG-TownCentre from a pedestrian zone. The average evaluation metrics achieved on the test sequences are given in Table 1, where they are compared to the related work (as of Dec. 2015). The reported metrics include the false alarms per frame (FAF), the ratio of mostly

\footnotetext{
${ }^{1}$ http://motchallenge. net
} 


\begin{tabular}{|l|l|l|l|l|l|l|l|l|l|l|l|}
\hline Method & Avg. Rank & MOTA & MOTP & FAF & MT & ML & FP & FN & IDs & Frag. & Hz \\
\hline GPDBN (ours) & 2.4 & 50.2 & $\mathbf{6 2 . 0}$ & 2.5 & $26.5 \%$ & $18.7 \%$ & 2252 & 5842 & $\mathbf{2 6 7}$ & $\mathbf{3 4 7}$ & 0.1 \\
\hline Klinger et al. (2015) & $\mathbf{2 . 0}$ & $\mathbf{5 1 . 1}$ & 61.0 & $\mathbf{2 . 3}$ & $\mathbf{2 8 . 7} \%$ & $17.9 \%$ & 2077 & $\mathbf{5 7 4 6}$ & 380 & 418 & 0.1 \\
\hline Leal-Taixé et al. (2011) & 3.1 & 35.9 & 54.0 & $\mathbf{2 . 3}$ & $13.8 \%$ & $21.6 \%$ & $\mathbf{2 0 3 1}$ & 8206 & 520 & 601 & 8.4 \\
\hline Baseline & 3.6 & 35.9 & 53.3 & 4.0 & $20.9 \%$ & $16.4 \%$ & 3588 & 6593 & 580 & 659 & $\mathbf{8 3 . 5}$ \\
\hline Pellegrini et al. (2009) & 3.9 & 25.0 & 53.6 & 3.6 & $6.7 \%$ & $\mathbf{1 4 . 6} \%$ & 3161 & 7599 & 1838 & 1686 & 30.6 \\
\hline
\end{tabular}

Table 1. 3D MOT 2015 results

tracked (MT, a person is MT if tracked for at least $80 \%$ of the time being present in consecutive images) and mostly lost (ML, if tracked for at most $20 \%$ ) objects, the numbers of false positive and false negative detections, the number of identity switches, the number of interruptions during the tracking of a person (Frag.), the processing frequency $(\mathrm{Hz}$, in frames per second) as well as MOTA and MOTP. The average rank shows the mean of the ranks achieved in the individual metrics.

As shown in Tab. 1, our method yields the best results in terms of geometric accuracy (MOTP), identity switches and interruptions, and an overall second best average rank of 2.4. Compared to the method of Klinger et al. (2015), on which our method is constructed, the results show that we have about $8 \%$ more false positive and $2 \%$ more false negative assignments. It is worth noting that although approximately the same rate of pedestrians could be tracked throughout their existence in the image sequence (as measured by the MT score), both the number of ID switches and interruptions could be reduced clearly about $30 \%$ and $16 \%$ compared to the work of Klinger et al. (2015), who do not take interactions into account. The benefit of using the implicit motion context as proposed in this paper can be attributed to the improved predictive model of the underlying recursive filter, in which the information of every tracked pedestrian is considered. In this way, even in the absence of observations a trajectory can be continued by exploiting the information about correlated (interacting) pedestrians. In such a case, a trajectory is only continued based on the predictions. As in a recursive filter the prior information (i.e., the previous state estimate) typically defines the search space for new target positions, an improved motion model yields fewer interruptions of trajectories. If motion context is not considered in the motion model, the prediction is prone to drift away from the target while at the same time being susceptible to an assignment to another target (causing an identity switch). Such a situation occurred in the exemplary scene depicted in Fig. 1 at the beginning of the paper.

The MOTP shows the normalised distance of a correct detection from the reference annotation in metres. According to that metric, the results of our method yield the highest geometric precision currently reported on the 3DMOT Benchmark (see Tab. 1, as of Dec. 2015). The MOTP value of 62.0 indicates that the average positional displacement of the automatically estimated pedestrian positions is about $38 \mathrm{~cm}$. This average offset basically stems from uncertainties at the conversion of the monocular image coordinates to the ground plane, which is assumed to be horizontal. Although the difference between the best (this) and the second best method in terms of that metric is only small, it shows that the covariance structure of pedestrians carries valuable information for the determination of the pedestrians' motion, and thus of the pedestrians' position. W.r.t. to processing time, this method, which is based on the same framework as Klinger et al. (2015), performs worst among the competing methods with only 0.1 frames per second. The prediction using the Gaussian Process Regression is as complex as the inversion of a $n \times n$ matrix (i.e., $\mathcal{O}\left(n^{3}\right)$ ), where $n$ is the number of pedestrians and only in the range of ten to thirty in our application scenario. Thus, the processing time of the algorithm is very similar to that of Klinger et al. (2015), where most of the computation time is used for the training of and classification with the online random forest classifier.

\section{CONCLUSIONS}

In this paper, we propose a new predictive model for a recursive Bayesian filter on the basis of Gaussian Process Regression. Using the proposed method, the state vector of a pedestrian is predicted from the states of all pedestrians being tracked. In this context, we propose a new covariance function which computes the covariance of two motion trajectories in terms of motion direction and spatial distance. The method is applied to a public benchmark dataset and the results show that the number of identity switches, the number of interruptions, and the geometric accuracy could be improved in comparison to the state-of-the-art. However, our tracking results also reveal an increase of false positive assignments, which are due to mismatches between the expected and the true motions. Such cases often occur during an occlusion, so that the trajectories are continued towards spurious destinations, causing false positive detections. To remedy such effects, a multimodal state representation will be investigated to evaluate both a prediction with and without using contextual information at the same time. When new measurements are obtained, the trajectory will be continued using the prediction that better coincides with the measurements.

\section{REFERENCES}

Bernardin, K. and Stiefelhagen, R., 2008. Evaluating multiple object tracking performance: the CLEAR MOT metrics. Journal on Image and Video Processing, vol. 2008, no. 1, pp. 1-10.

Choi, W. and Savarese, S., 2010. Multiple target tracking in world coordinate with single, minimally calibrated camera. In: Proc. European Conference on Computer Vision (ECCV), Springer, pp. 553-567.

Dalal, N. and Triggs, B., 2005. Histograms of oriented gradients for human detection. In: Proc. IEEE Conference on Computer Vision and Pattern Recognition (CVPR), pp. 886-893.

Ellis, D., Sommerlade, E. and Reid, I., 2009. Modelling pedestrian trajectory patterns with gaussian processes. In: Proc. IEEE International Conference on Computer Vision (ICCV) Workshop, pp. 1229-1234.

Ge, W., Collins, R. T. and Ruback, B., 2009. Automatically detecting the small group structure of a crowd. In: Proc. IEEE Workshop on Applications of Computer Vision (WACV), pp. 18 .

Helbing, D. and Molnár, P., 1995. Social force model for pedestrian dynamics. Physical review. E, Statistical Physics, Plasmas, Fluids, and Related Interdisciplinary Topics, 51 (5) pp. 42824286.

Hooke, R. and Jeeves, T. A., 1961. Direct search solution of numerical and statistical problems. Journal of the Association for Computing Machinery (JACM) 8(2), pp. 212-229. 
Kim, K., Lee, D. and Essa, I., 2011. Gaussian process regression flow for analysis of motion trajectories. In: Proc. IEEE International Conference on Computer Vision (ICCV), pp. 1164-1171.

Kim, K., Lee, D. and Essa, I., 2012. Detecting regions of interest in dynamic scenes with camera motions. In: Proc. IEEE Conference on Computer Vision and Pattern Recognition (CVPR), pp. $1258-1265$.

Klinger, T., Rottensteiner, F. and Heipke, C., 2015. Probabilistic multi-person tracking using dynamic Bayes networks. In: ISPRS Annals of the Photogrammetry, Remote Sensing and Spatial Information Sciences, Volume II-3/W5, pp. 435-442.

Ko, J. and Fox, D., 2009. GP-BayesFilters: Bayesian filtering using Gaussian process prediction and observation models. Autonomous Robots 27(1), pp. 75-90.

Krige, D. G., 1951. A statistical approach to some basic mine valuation problems on the Witwatersrand. Journal of Chemical, Metallurgical, and Mining Society of South Africa 94(3), pp. 95112 .

Leal-Taixé, L., Milan, A., Reid, I., Roth, S. and Schindler, K., 2015. MOTChallenge 2015: Towards a benchmark for multitarget tracking. arXiv:1504.01942 [cs].

Leal-Taixé, L., Pons-Moll, G. and Rosenhahn, B., 2011. Everybody needs somebody: Modeling social and grouping behavior on a linear programming multiple people tracker. In: Proc. IEEE International Conference on Computer Vision (ICCV) Workshop, pp. $120-127$.

Milan, A., Roth, S. and Schindler, K., 2014. Continuous energy minimization for multi-target tracking. IEEE Transactions on Pattern Analysis and Machine Intelligence, 36 (1) pp. 58-72.

Moritz, H., 1973. Least-Squares Collocation. German Geodetic Commission (DGK), Series A, Nr. 75, Munich, Germany.

Pellegrini, S., Ess, A. and Van Gool, L., 2010. Improving data association by joint modeling of pedestrian trajectories and groupings. In: Proc. European Conference on Computer Vision (ECCV), Springer, pp. 452-465.

Pellegrini, S., Ess, A., Schindler, K. and Van Gool, L., 2009. You'll never walk alone: Modeling social behavior for multitarget tracking. In: Proc. IEEE International Conference on Computer Vision (ICCV), pp. 261-268.

Rabiner, L. R., 1989. A tutorial on hidden Markov models and selected applications in speech recognition. Proceedings of the IEEE, 77 (2) pp. 257-286.

Rasmussen, C. E., 2006. Gaussian processes for machine learning. The MIT press.

Saffari, A., Leistner, C., Santner, J., Godec, M. and Bischof, H., 2009. On-line random forests. In: Proc. IEEE International Conference on Computer Vision (ICCV) Workshop, pp. 1393-1400.

Scovanner, P. and Tappen, M. F., 2009. Learning pedestrian dynamics from the real world. In: Proc. IEEE International Conference on Computer Vision (ICCV), pp. 381-388.

Urtasun, R., Fleet, D. J. and Fua, P., 2006. 3D people tracking with Gaussian process dynamical models. In: Proc. IEEE Conference on Computer Vision and Pattern Recognition (CVPR), Vol. 1, IEEE, pp. 238-245.

Yamaguchi, K., Berg, A. C., Ortiz, L. E. and Berg, T. L., 2011. Who are you with and where are you going? In: Proc. IEEE Conference on Computer Vision and Pattern Recognition (CVPR), pp. 1345-1352.
Yoon, J. H., Yang, M.-H., Lim, J. and Yoon, K.-J., 2015. Bayesian multi-object tracking using motion context from multiple objects. In: Proc. IEEE Winter Conference on Applications of Computer Vision (WACV), pp. 33-40.

Zhang, L. and van der Maaten, L., 2013. Structure preserving object tracking. In: Proc. IEEE Conference on Computer Vision and Pattern Recognition (CVPR), pp. 1838-1845. 